\title{
A Phase II Study to Evaluate the Safety and Efficacy of Pegteograstim in Korean Breast Cancer Patients Receiving Dose-Dense Doxorubicin/Cyclophosphamide
}

\author{
Gun Min Kim, MD ${ }^{1}$ \\ Joo Hoon Kim, MD' \\ Ji Heung Kim, MD \\ Young Up Cho, MD, PhD2 \\ Seung II Kim, MD, PhD² \\ Seho Park, MD, PhD² \\ Hyung Seok Park, MD² \\ Ji Ye Kim, MD2 \\ Joohyuk Sohn, MD, PhD
}

${ }^{1}$ Division of Medical Oncology,

Department of Internal Medicine,

${ }^{2}$ Department of Surgery, Yonsei University

College of Medicine, Seoul, Korea

Correspondence: Joohyuk Sohn, MD, PhD Division of Medical Oncology, Department of Internal Medicine, Yonsei University College of Medicine, 50-1 Yonsei-ro, Seodaemun-gu, Seoul 03722, Korea

Tel: 82-2-2228-8135

Fax: 82-2-393-3652

E-mail: oncosohn@yuhs.ac

Received June 30, 2018

Accepted September 18, 2018

Published Online September 19, 2018

\begin{abstract}
Purpose
Dose-dense chemotherapy (DD-CT) is a preferred (neo)adjuvant regimen in early breast cancer (BC). Although the results of reported randomized trials are conflicting, a recent metaanalysis showed improved overall and disease-free survival with DD-CT compared to conventional schedules. However, no DD-CT safety data for Korean BC patients are available. This phase II study was conducted to evaluate the safety and efficacy of pegteograstim in Korean $\mathrm{BC}$ patients receiving DD-CT.
\end{abstract}

\section{Materials and Methods}

Patients with operable (stage I-III), histologically confirmed BC received four cycles of intravenous doxorubicin $\left(60 \mathrm{mg} / \mathrm{m}^{2}\right)$ and cyclophosphamide $\left(600 \mathrm{mg} / \mathrm{m}^{2}\right)$ on day 1 every 2 weeks as neoadjuvant or adjuvant therapy. Pegteograstim $(6.0 \mathrm{mg})$ was administered subcutaneously on day 2 of each cycle. The primary endpoint was the incidence of febrile neutropenia (FN). The secondary endpoints were safety and tolerability.

\section{Results}

Of 63 patients, one (1.6\%) developed FN during all cycles of DD-CT. Dose delay was observed in four patients (6.3\%) and dose reduction in two (3.2\%) during DD-CT. Frequent adverse events (AEs) were nausea, alopecia, generalized muscle weakness, myalgia, mucositis, anorexia, dyspepsia, and diarrhea; most AEs were related to chemotherapy. Grade 3-4 AEs were reported in five of 63 patients (7.9\%), and all grade 3 and 4 AEs were related to chemotherapy. Adverse drug reactions possibly linked to pegteograstim were abdominal pain, bone pain, myalgia, generalized muscle weakness, and headache in five of 63 patients (7.9\%).

\section{Conclusion}

Dose-dense AC (doxorubicin/cyclophosphamide) chemotherapy with pegteograstim support is a tolerable and safe regimen in Korean early $\mathrm{BC}$ patients.

\section{Introduction}

Anthracycline followed by taxane chemotherapy regimens are considered the standard of care as neoadjuvant or adjuvant chemotherapy for early breast cancer [1]. The introduction of granulocyte colony stimulating factor (G-CSF) enabled dose-dense chemotherapy (DD-CT), defined as the administration of the standard dose chemotherapy over shorter intervals per cycle. The National Comprehensive
Key words

Breast neoplasms, Dose-dense chemotherapy, Pegfilgrastim
Cancer Network guidelines recommend a DD regimen as the preferred treatment based on the results of several randomized trials that showed the superiority of DD-CT [2-4]. A recent meta-analysis also reported that DD-CT with G-CSF support lowers the risk of recurrence and breast cancer death by approximately $15 \%$ in cases of early breast cancer [5].

However, along with the increase in efficacy, DD-CT has the potential to increase toxicity. Several prospective DD-CT trials reported that DD-CT was associated with an increased rate of non-hematologic and hematologic toxicities [2,3,6-9]. 
Waks et al. [10] reported that the DD-CT regimen was a risk factor for Pneumocystis jirovecii pneumonia (PJP) in a retrospective review of a large population $(n=3,322)$. In addition, limited safety data of DD-CT in Asian breast cancer patients are available, and several studies have shown that the incidence of chemotherapy-related toxicities differs between ethnic groups [11-15]. An investigation of the safety profiles of DD-CT with long-acting G-CSF in Asian breast cancer patients is needed.

Pegteograstim, a novel long-acting recombinant human G-CSF, has higher biological activity based on its higher affinity to the G-CSF receptor than pegfilgrastim [16-18]. Here, we evaluated the safety and efficacy of pegteograstim in Korean early breast cancer patients receiving DD-AC (doxorubicin/cyclophosphamide) regimen.

\section{Materials and Methods}

\section{Study design and patients}

This was a single arm, phase II trial conducted at Yonsei Cancer Center in Korea.

Eligible patients were women with histologically confirmed, operable primary breast cancer without distant metastases. The planned regimen was AC (doxorubicin 60 $\mathrm{mg} / \mathrm{m}^{2}$ and cyclophosphamide $600 \mathrm{mg} / \mathrm{m}^{2}$ ) chemotherapy as neoadjuvant or adjuvant therapy for breast cancer. Patients had to have an Eastern Cooperative Oncology Group (ECOG) Performance Status score of 0 or 1 and no major cardiovascular morbidity or other serious medical conditions. The main exclusion criteria were previous adjuvant or neoadjuvant treatment, ongoing pregnancy or lactation, abnormal laboratory values precluding the possibility to safely deliver the cytotoxic agents used in the study, and previous or concurrent malignant neoplasms at the other sites.

\section{Procedures}

Patients received four cycles of DD-AC $\left(60 \mathrm{mg} / \mathrm{m}^{2}\right.$ doxorubicin and $600 \mathrm{mg} / \mathrm{m}^{2}$ cyclophosphamide administered intravenously on day 1 every 2 weeks). A fixed dose of 6.0 mg pegteograstim was administered subcutaneously on day 2 of each chemotherapy cycle (between 22 and 26 hours after the completion of chemotherapy). Patients with lymph node metastases were treated with taxane chemotherapy (weekly paclitaxel or docetaxel every 3 weeks). Taxane regimen was decided by investigator's discretion.

Blood sampling for hematological and biochemical assessments was done on day 1 of each cycle and 4 weeks after the last chemotherapy (end of treatment visit). Chemotherapy was administered only if absolute neutrophil count was $\geq 1.0 \times 10^{3}$ cells / L and platelet counts were $\geq 100 \times 10^{3} / \mathrm{L}$. Supportive care was provided as per the local policy. A dose delay of up to 14 days was allowed to achieve these hematologic parameters. Adverse events (AEs) were graded using the National Cancer Institute Common Terminology Criteria for Adverse Events (NCI-CTCAE ver. 4.03).

\section{Outcomes}

The primary endpoint was the incidence of febrile neutropenia (FN) in DD-AC with pegteograstim. FN was defined as neutropenia $(<500$ neutrophils $/ \mu \mathrm{L})$ with a febrile event (a single oral temperature of $\geq 38.3^{\circ} \mathrm{C}$ or a temperature of $\geq 38.0^{\circ} \mathrm{C}$ sustained over a 1-hour period). Secondary endpoints were the incidences of $\mathrm{FN}$ in the first cycle of DD-AC, hospitalization for $\mathrm{FN}$, grade 3 or 4 neutropenia in the first cycle of DD-AC, and the dose delay or reduction of chemotherapy. Safety was also a secondary endpoint and was assessed throughout the study treatment according to the NCI-CTCAE ver. 4.03.

\section{Statistical considerations}

In previous DD-CT trials, the incidence of FN in DD-AC ranged widely from $1 \%$ to $10 \%$ and several guidelines categorize the $\mathrm{AC}$ regimen into the intermediate risk group (risk of FN ranges from 10\%-25\%). Based on those results, we hypothesized that threshold and expected values of the incidences of FN were $5 \%$ and $20 \%$, respectively. To meet the threshold and expected values of the incidence of FN with a type I error level of 0.05 and type II error level of 0.20 , at least 56 patients were required. As a dropout rate of $10 \%$ is expected, we aimed to recruit 63 patients. Statistical analyses were performed using SPSS ver. 22.0 for Window (IBM Corp., Armonk, NY). Descriptive statistics were used for baseline characteristics. Binomial two-sided $95 \%$ confidence intervals (CIs) for the change of hematology profile were calculated.

\section{Ethical statement}

All patients provided written informed consent before enrollment. The study protocol was approved by the institutional review board (IRB No. 4-2015-0813). The study was conducted according to the Declaration of Helsinki and the principles of good clinical practice. The trial was registered at ClinicalTrials.gov (NCT03575520). 
Table 1. Baseline characteristics

\begin{tabular}{|c|c|}
\hline & No. $(\%)(n=63)$ \\
\hline Age, median (range, yr) & $50(29-69)$ \\
\hline \multicolumn{2}{|l|}{ Menopausal status } \\
\hline Premenopausal & $42(66.7)$ \\
\hline Postmenopausal & $21(33.3)$ \\
\hline \multicolumn{2}{|l|}{ Type of surgery } \\
\hline Mastectomy & $23(36.5)$ \\
\hline Breast-conserving surgery & $40(63.5)$ \\
\hline \multicolumn{2}{|l|}{ T category } \\
\hline $\mathrm{T} 1$ & $26(41.3)$ \\
\hline $\mathrm{T} 2$ & $28(44.4)$ \\
\hline T3 & $9(14.3)$ \\
\hline \multicolumn{2}{|l|}{$\mathrm{N}$ category } \\
\hline 0 & $26(41.3)$ \\
\hline 1 & $28(44.4)$ \\
\hline 2 & $4(6.3)$ \\
\hline 3 & $5(7.9)$ \\
\hline \multicolumn{2}{|l|}{ Tumor grade } \\
\hline 1 & $3(4.8)$ \\
\hline 2 & $35(55.6)$ \\
\hline 3 & $22(34.9)$ \\
\hline Missing & $3(4.8)$ \\
\hline \multicolumn{2}{|l|}{ TNM stage } \\
\hline 1 & $29(46.0)$ \\
\hline 2 & $28(44.4)$ \\
\hline 3 & $6(9.5)$ \\
\hline \multicolumn{2}{|l|}{ Subtype } \\
\hline HR+ / HER2- & $32(50.8)$ \\
\hline HR + / HER2+ & $5(7.9)$ \\
\hline HR-/HER2+ & $15(23.8)$ \\
\hline HR-/HER2- & $11(17.5)$ \\
\hline \multicolumn{2}{|l|}{ Treatment setting } \\
\hline Neoadjuvant & $18(28.6)$ \\
\hline Adjuvant & $45(71.4)$ \\
\hline
\end{tabular}

TNM, tumor node metastasis; HR, hormone receptor; HER2, human epidermal growth factor receptor 2.

\section{Results}

\section{Patient characteristics}

Between June 2016 and March 2017, a total of 63 patients were enrolled at Yonsei Cancer Center. The characteristics of the patients included in this study are shown in Table 1 . The median (range) age was 50 years (range, 29 to 69 years), and all patients had an ECOG performance status score of 0 . Of the 63 patients, 42 (66.7\%) were premenopausal women, and 37 (58.7\%) were node positive, $22(24.9 \%)$ had grade 3 dis- ease, $18(28.6 \%)$ received neoadjuvant therapy, 37 (58.7\%) had estrogen receptor- or progesterone receptor-positive tumors, and $20(31.7 \%)$ had human epidermal growth factor receptor 2-positive tumors.

\section{The incidence of FN and dose intensity}

Only one of 63 patients (1.6\%) developed FN during DD-AC chemotherapy. All patients, except one who withdrew from the study after the first cycle of chemotherapy, completed the planned four cycles of DD-AC chemotherapy. Dose delay was observed in four of 63 patients $(6.3 \%)$ during DD-AC. The duration of dose delay was seven days for all four patients. The reasons for the dose delay were AEs in two patients (liver enzyme elevation grade 3 and mucositis grade 2). The other two patients visited 1 week later due to a mistaken appointment without any reason for the dose delay. Dose reduction was observed in two of 63 patients (3.2\%) during DD-AC. The reasons for the dose reductions were recurrent grade $3 \mathrm{FN}$ and grade 3 mucositis in each patient.

\section{Safety}

The most frequently reported AEs are presented in Table 2. All but one of the 63 patients $(98.4 \%)$ reported at least one AE during DD-AC chemotherapy with pegteograstim subcutaneous injection. Grade 3 to 4 AEs were reported in five of 63 patients $(7.9 \%)$. All grade 3 or 4 AEs were linked to chemotherapy (grade $3 \mathrm{FN}$, grade 3 anemia, grade 3 pneumonia, grade 3 mucositis, grade 3 wound infection, and grade 4 neutropenia). One patient withdrew from the study after the first cycle of chemotherapy due to toxicity (pneumonia). She refused further adjuvant chemotherapy. The most frequent AEs were nausea, alopecia, generalized muscle weakness, myalgia, mucositis, anorexia, dyspepsia, diarrhea, and most AEs were due to the chemotherapy. Adverse drug reactions (ADRs) that might be linked to pegteograstim included abdominal pain, bone pain, myalgia, generalized muscle weakness, and headache in five of 63 patients (7.9\%). Ten patients experience infectious AEs. Regarding to the type of infections, cystitis is most common with six cases $(60 \%)$ and the remainder were pneumonia, febrile neutropenia, enteritis, esophageal candidiasis, respectively.

\section{Change of hematology profile}

The change in hematology profiles (neutrophil, lymphocyte, platelet counts, and hemoglobin level) at day 1 of each cycle and follow-up is shown in Fig. 1. Mean absolute lymphocyte counts and hemoglobin level decreased gradually during DD-AC chemotherapy and at the follow-up visit. Mean platelet counts decreased gradually during DD-AC 
Table 2. Adverse events

\begin{tabular}{|c|c|c|c|}
\hline Adverse event & $\begin{array}{l}\text { All grades } \\
\qquad(n=63)\end{array}$ & $\begin{array}{c}\text { Grade 1-2 } \\
\quad(n=63)\end{array}$ & $\begin{array}{l}\text { Grade 3-4 } \\
\quad(n=63)\end{array}$ \\
\hline \multicolumn{4}{|l|}{ Hematologic } \\
\hline Neutropenia & $12(19)$ & $10(16)$ & $2(3)$ \\
\hline Anemia & $56(89)$ & $54(86)$ & $2(3)$ \\
\hline Thrombocytopenia & $8(13)$ & $8(13)$ & 0 \\
\hline Lymphopenia & $50(79)$ & $41(65)$ & $9(14)$ \\
\hline Febrile neutropenia & $1(2)$ & - & $1(2)$ \\
\hline \multicolumn{4}{|l|}{ Non-hematologic } \\
\hline Infection & $10(16)$ & $8(13)$ & $2(3)$ \\
\hline Alopecia & $27(43)$ & $27(43)$ & 0 \\
\hline Asthenia & $25(40)$ & $25(40)$ & 0 \\
\hline Anorexia & $15(24)$ & $15(24)$ & 0 \\
\hline Nausea & $37(59)$ & $37(59)$ & 0 \\
\hline Vomiting & $2(3)$ & $2(3)$ & 0 \\
\hline Stomatitis & $24(38)$ & $23(37)$ & $1(2)$ \\
\hline Diarrhea & $10(16)$ & $10(16)$ & 0 \\
\hline Constipation & $11(17)$ & $11(17)$ & 0 \\
\hline Bone pain & $8(13)$ & $8(13)$ & 0 \\
\hline Myalgia & $22(35)$ & $22(35)$ & 0 \\
\hline Pain, others & $34(54)$ & $34(54)$ & 0 \\
\hline Cough & $17(27)$ & $17(27)$ & 0 \\
\hline Dizziness & $21(33)$ & $21(33)$ & 0 \\
\hline Dyspepsia & $25(40)$ & $25(40)$ & 0 \\
\hline Insomnia & $15(24)$ & $15(24)$ & 0 \\
\hline Transaminase elevation & $28(44)$ & $28(44)$ & 0 \\
\hline Others & $38(60)$ & $38(60)$ & 0 \\
\hline
\end{tabular}

Values are presented as number (\%).

chemotherapy but recovered to baseline levels at follow-up. In contrast, mean neutrophil counts were increased during DD-AC but subsequently returned to baseline level at follow-up.

\section{Discussion}

To the best of our knowledge, this study is the first to evaluate the efficacy and safety of long acting G-CSF, pegteograstim during DD-AC chemotherapy in Korean early breast cancer patients. We found that FN occurred in only $1.6 \%$ of patients receiving DD-AC chemotherapy with pegteograstim support. Grade 3 to 4 AEs were reported in five of 63 patients $(7.9 \%)$. All but one patient completed the planned four cycles of DD-AC chemotherapy. Dose delay and dose reduction linked to AEs were observed in two of 63 patients (3.2\%). The most common ADRs possibly linked to pegteograstim were abdominal pain, bone pain, myalgia, generalized muscle weakness, and headache.

CALGB 9741 trial was the first large phase III study to demonstrate that DD-CT improved clinical outcomes regarding both disease-free survival (DFS) and overall survival (OS) in node-positive breast cancer [3]. Similarly, the GIM-II trial showed that DD-CT improved DFS and OS compared to a conventional chemotherapy schedule. In contrast, several trials exploring the role of DD-CT have reported negative results [6-8]. The most recent meta-analysis by the Early Breast Cancer Trialists' Collaborative Group found that DDCT lowers the risk of recurrence and breast cancer death by approximately $15 \%$ in early breast cancer [5]. In Korea, longacting G-CSF is now available, but DD-CT is not commonly used as is the case in Western settings. The reasons for this are a lack of safety data for DD-CT in Korean patients and non-reimbursement of long-acting G-CSF for DD-CT.

DD-CT may increase most chemotherapy-related toxicities. In individual trials, non-hematologic and hematologic AEs, except neutropenia, were higher for DD-CT than for 
A
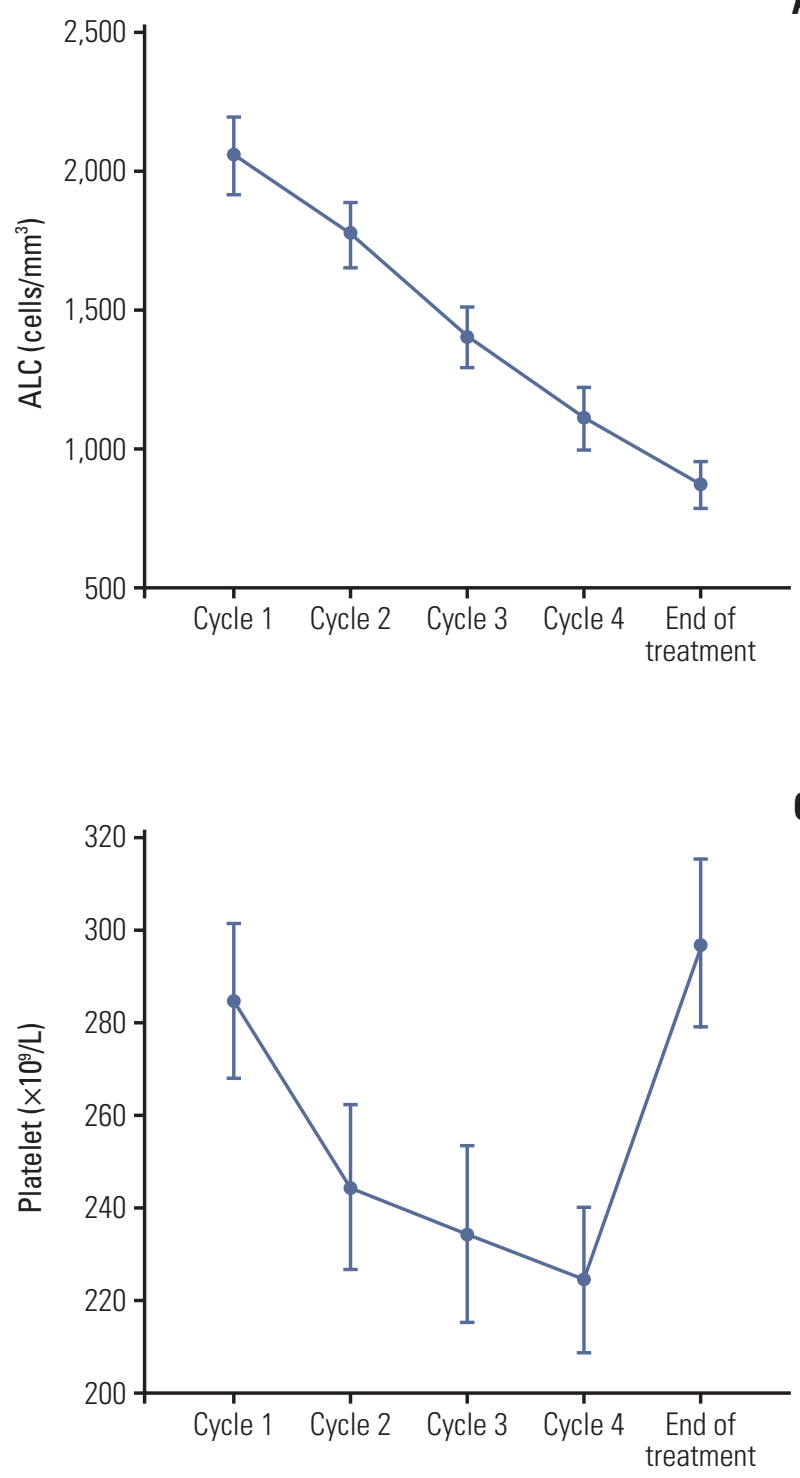

C
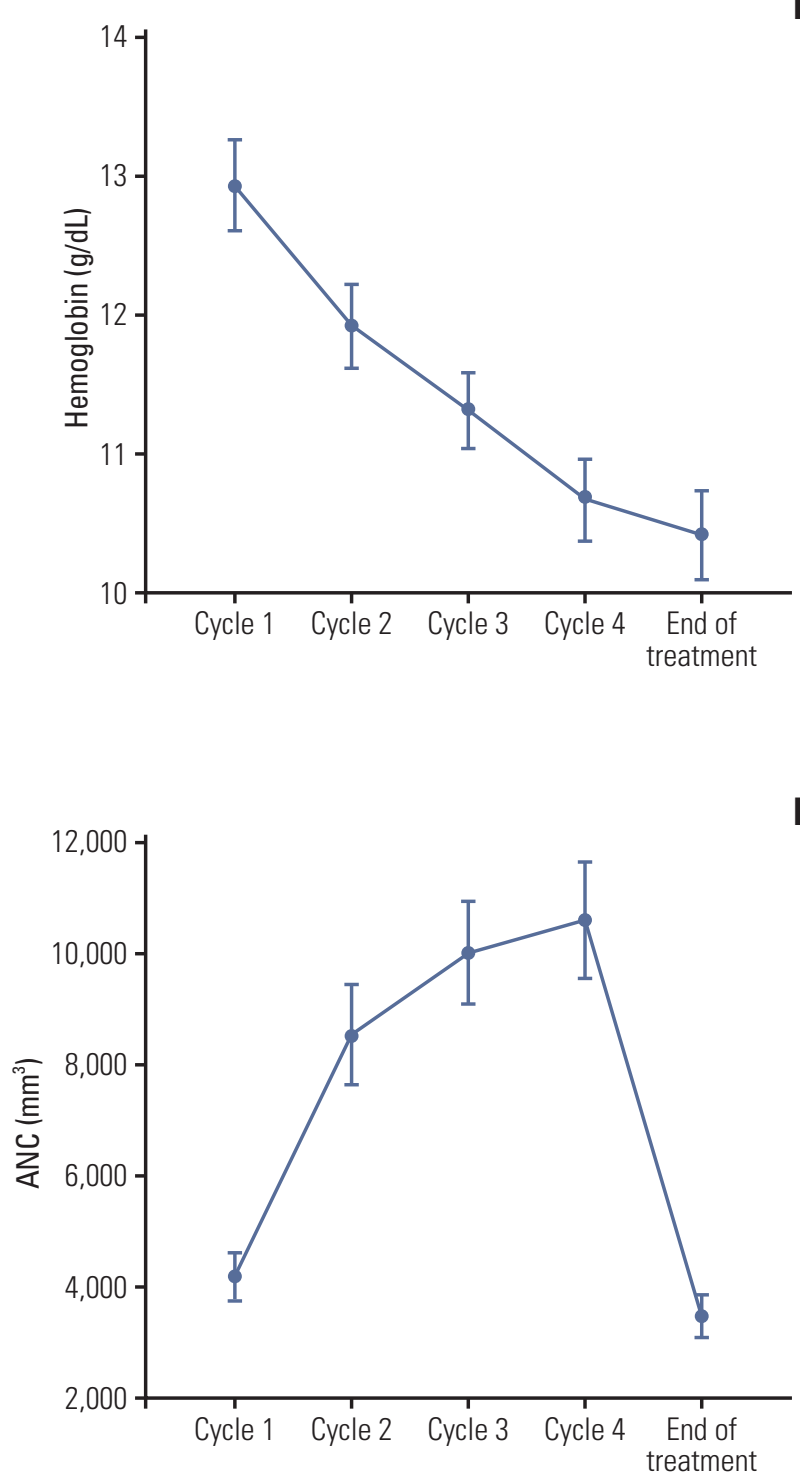

Fig. 1. Change in hematology profiles on day 1 of each cycle (cycle 1 to 4 ) and end of treatment (4 weeks after the last chemotherapy). (A) Absolute lymphocyte count (ALC). (B) Hemoglobin level. (C) Platelet count. (D) Absolute neutrophil count (ANC).

conventional schedule chemotherapy [2,6-9]. Petrelli et al. [19] reported that grade 3 to 4 neutropenia and FN rates were not different between DD-CT and conventional chemotherapy, but rates of anemia and thrombocytopenia were significantly higher (relative risk [RR], 3.48; 95\% CI, 2.89 to 4.2, $\mathrm{p}<0.001$ and $\mathrm{RR}, 2.85 ; 95 \% \mathrm{CI}, 1.93$ to $4.2, \mathrm{p}<0.001$ ) during DD-CT than during conventional chemotherapy [19]. In this study, the incidence of grade 3 or 4 AEs, including FN $(1.6 \%)$, was within an acceptable range and there were no toxicityrelated deaths. At least in terms of frequency of FN, DD-AC with G-CSF support was safer than conventional AC chemotherapy without G-CSF use.

Pegteograstim is a novel monoPEGylated recombinant human G-CSF. It displays higher biological activity based on an improved binding affinity to the G-CSF receptor [16-18]. Lee et al. [18] showed that pegteograstim was as effective as pegfilgrastim in the reduction of chemotherapy-induced neutropenia in breast cancer patients; the incidence of ADRs was 5.3\%, and most common ADRs were leukocytosis, blurred vision, injection site pain, headache, and dizziness 
$[16,17]$. In this study, the rate of ADRs for pegteograstim was 7.9\% with AE profiles similar to the previous study.

We investigated the change in neutrophil, lymphocyte, platelet, and hemoglobin level at every visit during DD-AC chemotherapy (Fig. 1). Interestingly, lymphocyte count and hemoglobin levels were not recovered one month after the completion of chemotherapy. Although there was no case of PJP in this study, the DD-AC regimen was one of the risk factors for PJP in the retrospective study by Waks et al. [10] They suggested that a high-dose steroid used as an antiemetic over 8 weeks may increase the PJP risk in DD-AC. In this study, we found that most patients had lymphopenia after completion of DD-AC. Prolonged lymphopenia can contribute to infectious vulnerability for PJP.

This study has several limitations. First, this was a smallsized single-center study. Second, this study did not collect patient-reported outcome (PRO) data. PRO data are increasingly being implemented in oncology clinical trials to evaluate disease-related symptoms, treatment-related AEs, and health-related impacts on quality of life. We collected $\mathrm{AE}$ information from patients at regular visits and therefore $\mathrm{AE}$ information may not be collected thoroughly. Third, $28.6 \%$ of patients received DD-AC as neoadjuvant therapy in this study. There is a possibility of different toxicity profiles between neoadjuvant therapy and adjuvant therapy, despite the regimens being identical.

In conclusion, DD-AC chemotherapy with pegteograstim support is an efficacious and safe regimen in Korean patients with early breast cancer. Reimbursement by the Korean government of long-acting G-CSF used for DD-CT is warranted in early breast cancer patients.

\section{Conflicts of Interest}

Pegteograstim was supplied by the GC Pharma.

\section{Acknowledgments}

This research was supported by a grant of the Korea Health Technology R\&D Project through the Korea Health Industry Development Institute (KHIDI), funded by the Ministry of Health \& Welfare, Republic of Korea (grant number: HI14C1324).

\section{References}

1. Early Breast Cancer Trialists' Collaborative Group, Peto R, Davies C, Godwin J, Gray R, Pan HC, et al. Comparisons between different polychemotherapy regimens for early breast cancer: meta-analyses of long-term outcome among 100,000 women in 123 randomised trials. Lancet. 2012;379:432-44.

2. Del Mastro L, De Placido S, Bruzzi P, De Laurentiis M, Boni C, Cavazzini G, et al. Fluorouracil and dose-dense chemotherapy in adjuvant treatment of patients with early-stage breast cancer: an open-label, $2 \times 2$ factorial, randomised phase 3 trial. Lancet. 2015;385:1863-72.

3. Citron ML, Berry DA, Cirrincione C, Hudis C, Winer EP, Gradishar WJ, et al. Randomized trial of dose-dense versus conventionally scheduled and sequential versus concurrent combination chemotherapy as postoperative adjuvant treatment of node-positive primary breast cancer: first report of Intergroup Trial C9741/Cancer and Leukemia Group B Trial 9741. J Clin Oncol. 2003;21:1431-9.

4. National Comprehensive Cancer Network. NCCN clinical practice guidelines in oncology: breast cancer. Plymouth Meeting, PA: National Comprehensive Cancer Network; 2018.

5. Gray R, Bradley R, Braybrooke J, Davies C, Pan H, Peto R, et al. Increasing the dose density of adjuvant chemotherapy by shortening intervals between courses or by sequential drug administration significantly reduces both disease recurrence and breast cancer mortality: an EBCTCG meta-analysis of 21,000 women in 16 randomised trials. In: San Antonio Breast Cancer Symposium; 2017 Dec 5-9; San Antonio, TX, USA.
Philadelphia, PA: American Association for Cancer Research; 2018.

6. Venturini M, Del Mastro L, Aitini E, Baldini E, Caroti C, Contu $\mathrm{A}$, et al. Dose-dense adjuvant chemotherapy in early breast cancer patients: results from a randomized trial. J Natl Cancer Inst. 2005;97:1724-33.

7. Foukakis T, von Minckwitz G, Bengtsson NO, Brandberg Y, Wallberg B, Fornander T, et al. Effect of tailored dose-dense chemotherapy vs standard 3-weekly adjuvant chemotherapy on recurrence-free survival among women with high-risk early breast cancer: a randomized clinical trial. JAMA. 2016; 316:1888-96.

8. Cameron D, Morden JP, Canney P, Velikova G, Coleman R, Bartlett J, et al. Accelerated versus standard epirubicin followed by cyclophosphamide, methotrexate, and fluorouracil or capecitabine as adjuvant therapy for breast cancer in the randomised UK TACT2 trial (CRUK/05/19): a multicentre, phase 3, open-label, randomised, controlled trial. Lancet Oncol. 2017;18:929-45.

9. Mobus V, Jackisch C, Luck HJ, du Bois A, Thomssen C, Kuhn $\mathrm{W}$, et al. Ten-year results of intense dose-dense chemotherapy show superior survival compared with a conventional schedule in high-risk primary breast cancer: final results of AGO phase III iddEPC trial. Ann Oncol. 2018;29:178-85.

10. Waks AG, Tolaney SM, Galar A, Arnaout A, Porter JB, Marty $\mathrm{FM}$, et al. Pneumocystis jiroveci pneumonia (PCP) in patients receiving neoadjuvant and adjuvant anthracycline-based 
chemotherapy for breast cancer: incidence and risk factors. Breast Cancer Res Treat. 2015;154:359-67.

11. Gandara DR, Kawaguchi T, Crowley J, Moon J, Furuse K, Kawahara M, et al. Japanese-US common-arm analysis of paclitaxel plus carboplatin in advanced non-small-cell lung cancer: a model for assessing population-related pharmacogenomics. J Clin Oncol. 2009;27:3540-6.

12. Hasegawa Y, Kawaguchi T, Kubo A, Ando M, Shiraishi J, Isa $\mathrm{S}$, et al. Ethnic difference in hematological toxicity in patients with non-small cell lung cancer treated with chemotherapy: a pooled analysis on Asian versus non-Asian in phase II and III clinical trials. J Thorac Oncol. 2011;6:1881-8.

13. Wang Y, Choueiri TK, Lee JL, Tan MH, Rha SY, North SA, et al. Anti-VEGF therapy in mRCC: differences between Asian and non-Asian patients. Br J Cancer. 2014;110:1433-7.

14. Huang RS, Kistner EO, Bleibel WK, Shukla SJ, Dolan ME. Effect of population and gender on chemotherapeutic agentinduced cytotoxicity. Mol Cancer Ther. 2007;6:31-6.

15. Kim CG, Sohn J, Chon H, Kim JH, Heo SJ, Cho H, et al. Incidence of febrile neutropenia in Korean female breast cancer patients receiving preoperative or postoperative doxorubicin/ cyclophosphamide followed by docetaxel chemotherapy. J Breast Cancer. 2016;19:76-82.

16. Hong J, Lee B, Kang K, Lee SH, Ryu J, Jung G, et al. Characterisation of the site-specific monoPEGylated rhG-CSF analogue pegteograstim. Biologicals. 2018;51:54-61.

17. Shin KH, Lim KS, Lee H, Jang IJ, Yu KS. An assessment of the pharmacokinetics, pharmacodynamics, and tolerability of GCPGC, a novel pegylated granulocyte colony-stimulating factor (G-CSF), in healthy subjects. Invest New Drugs. 2014;32: 636-43.

18. Lee KH, Kim JY, Lee MH, Han HS, Lim JH, Park KU, et al. A randomized, multicenter, phase II/ III study to determine the optimal dose and to evaluate the efficacy and safety of pegteograstim (GCPGC) on chemotherapy-induced neutropenia compared to pegfilgrastim in breast cancer patients: KCSG PC10-09. Support Care Cancer. 2016;24:1709-17.

19. Petrelli F, Cabiddu M, Coinu A, Borgonovo K, Ghilardi M, Lonati V, et al. Adjuvant dose-dense chemotherapy in breast cancer: a systematic review and meta-analysis of randomized trials. Breast Cancer Res Treat. 2015;151:251-9. 